\title{
Methods for precise molecular detection of probiotic microflora : using adjusted molecular biology protocols, primer sets and PCR assays.
}

\begin{abstract}
Lactobacillus sp. is probiotic bacteria for which many detection methods were envisaged. However, culture-based methods failed to achieve specific detection of this bacterium due to its presence in mixed bacterial complex communities. The PCR assay was optimized to detect and quantify Lactobacillus sp. specifically in complex microbial community of mixed bacteria. Four DNA extraction methods, DNA integrity, primers specificity and optimized PCR procedure were all tested. It was shown that extracted genomic DNA using Wizard ${ }^{\circledR}$ Genomic DNA Purification Kit showed the highest yield, quality and performance in gel electrophoresis. Moreover, the specificity of the primer set, Lacto-16S-F /Lacto-16S-R, specific for Lactobacillus sp. was checked and found highly specific. In conclusion, the best DNA extraction protocol, working specific primer set and working PCR assay were achieved for achieving efficient, specific and reliable molecular-based, culture-independent, method of detection of lactobacillus sp. in PCR-suppressor highly protein-complex environment of mixed bacteria community.
\end{abstract}

Keyword: PCR; DNA extraction; Lactobacillus sp.; Probiotics. 